\title{
Algorithm for Identifying Minimum Driver Nodes Based on Structural Controllability
}

\author{
Reza Haghighi' ${ }^{1}$ and HamidReza Namazi ${ }^{2}$ \\ ${ }^{1}$ Department of Electrical and Computer Engineering, National University of Singapore, Singapore 119077 \\ ${ }^{2}$ Department of Mechanical Engineering, Faculty of Engineering, Universiti Malaysia Sarawak, 94300 Kuching, Malaysia \\ Correspondence should be addressed to HamidReza Namazi; m080012@e.ntu.edu.sg
}

Received 15 May 2015; Accepted 30 July 2015

Academic Editor: Hou-Sheng Su

Copyright (c) 2015 R. Haghighi and H. Namazi. This is an open access article distributed under the Creative Commons Attribution License, which permits unrestricted use, distribution, and reproduction in any medium, provided the original work is properly cited.

Existing methods on structural controllability of networked systems are based on critical assumptions such as nodal dynamics with infinite time constants and availability of input signals to all nodes. In this paper, we relax these assumptions and examine the structural controllability for practical model of networked systems. We explore the relationship between structural controllability and graph reachability. Consequently, a simple graph-based algorithm is presented to obtain the minimum driver nodes. Finally, simulation results are presented to illustrate the performance of the proposed algorithm in dealing with large-scale networked systems.

\section{Introduction}

Advances in communications technology have opened up new challenges in the area of networked systems. Controllability of multiagent networked systems as a fundamental concept in this field has received considerable attention. The pioneer work in analysing controllability of multiagent systems with leader-follower architecture had been carried out by Tanner [1], where controllability conditions were provided for multiagent systems with undirected graph topology based on eigenvectors of the Laplacian matrix. In further development, some algebraic conditions for controllability of multiagent systems are presented in $[2,3]$. Ji and Egerstedt [4] introduced network equitable partitions to present a necessary condition for the controllability of leaderfollower multiagent systems. Inspired by [4], Rahmani et al. [5] proposed the controllability of multiagent systems with multiple leaders. Liu et al. [6] derived a simple controllability condition for discrete-time single-leader switching networks, which was further extended to continuous-time single-leader switching networks [7]. Ji et al. [8] derived a necessary and sufficient condition for the controllability of leaderfollower multiagent systems, by dividing the overall system into several connected components. The other related topics in this area are leader-follower consensus $[9,10]$, leaderfollower formation control [11-13], containment control [14, $15]$, and pinning-controllability of networked systems $[16,17]$.

The concept of structural controllability has been studied extensively since the classical work by Lin [18]. In [18], structural controllability of SISO linear systems was explored by introducing a notion of structured matrix whose elements are either fixed zeros or independent free parameters. Shields and Pearson [19] extended the results of [18] to structural controllability of multiinput linear systems. Since then various works have been carried out on the structural controllability of linear systems [20-22]. Recently, structural controllability of networked systems has emerged as a major interest in the network sciences. A notable work in this area is carried out by Liu et al. [23] which addressed the structural controllability of complex networks. Jafari et al. [24] studied structural controllability of a leader-follower multiagent system with multiple leaders. Sundaram and Hadjicostis [25] developed a graph-theoretic characterization of controllability and observability of linear systems over finite fields. Haghighi and Cheah [26] employed the concept of structural observability to examine the weight-balanceability of networked systems. 
For large-scale networked systems, it is infeasible to apply input signals to all network nodes due to the high control cost and the difficulty of practical implementations. In this case, a fundamental problem is to identify a certain amount of nodes to be driven externally to bring the whole network under control. This problem was addressed in [23], where a theoretical framework was developed to solve the minimum input problem based on Lin's structural controllability theorem [18]. As pointed out in [27], the results in [23] are based on the assumption that each node has an infinite time constant, which do not generally represent the dynamics of the physical and biological systems.

Despite the model in [23], Cowan et al. [27] considered internal dynamics for all nodes of the network. Cowan's result states that structural controllability does not depend on degree distribution. Hence, the structural controllability can always be conferred with a single independent control input. However, the result in [27] suffers from a drawback that each independent input is connected to all nodes in the network, which is practically infeasible.

In this paper, we examined the structural controllability in networked systems by relaxing the critical assumptions in previous results. Consequently, we provide a graph-theoretic method to identify driver nodes. We present an algorithm to determine minimum driver nodes in networked systems. The contribution of this paper is twofold: (i) we relaxed the assumptions in existing methods, such as infinite time constant for each node, and having direct access to input signals by all nodes, on structural controllability of networked systems. (ii) We provide a simple algorithm to obtain minimum driver nodes in networked systems.

The paper is organized as follows. Section 2 presents some preliminaries in graph theory and controllability. Section 3 presents the model of the networked systems. Section 4 addresses the structural controllability in networked systems and presents the linkage between the structural controllability and the graph reachability in networked systems. An algorithm for identifying minimum driver nodes in networked systems is proposed in Section 5. Section 6 presents the simulation results and Section 7 concludes this paper.

\section{Preliminaries}

The communication between nodes can be expressed by a weighted directed graph $\mathscr{G}(\mathscr{V}, \mathscr{E}, \mathscr{A})$, such that $\mathscr{V}=$ $\left\{v_{1}, v_{2}, \ldots, v_{N}\right\}$ represents the set of nodes, $\mathscr{E} \subseteq \mathscr{V} \times \mathscr{V}$ is the edge set, and $\mathscr{A}=\left[a_{i j}\right]$ is the weighted adjacency matrix where $a_{i j}>0$ if $(i, j) \in \mathscr{E}$ and $a_{i j}=0$ otherwise. A graph $\mathscr{H}$ is said to be a subgraph of a graph $\mathscr{G}$ if $\mathscr{V}(\mathscr{H}) \subseteq \mathscr{V}(\mathscr{G})$ and $\mathscr{E}(\mathscr{H}) \subseteq \mathscr{E}(\mathscr{G})$

A directed path in a digraph is an ordered sequence of nodes so that any two consecutive nodes in the sequence are an edge of the digraph. An undirected graph is a tree if and only if, for any two nodes, there is a unique path connecting them. A directed spanning tree or arborescence is a digraph such that there is a unique directed path from a designated root node to every other node.
Definition 1. A digraph $\mathscr{G}$ is called an arborescence diverging from node $u$, if there is only one directed path between root $u$ and any other node of $\mathscr{G}$. If $\mathscr{G}$ is an arborescence diverging from $u$, then its reverse digraph (i.e., all edges of $\mathscr{G}$ are reversed) is called an arborescence converging to $u$ [28].

If there is an arborescence subdigraph diverging from an arbitrary node $u$, then $u$ is called a globally reachable node.

Definition 2. Driver nodes are nodes in a network that have to be controlled in order to completely control the entire network.

Definition 3. A matrix $A_{s}$ is said to be a structured matrix if its elements are either fixed zeros or independent free parameters [29].

Definition 4. Two dynamical systems are called structurally equivalent, if their interconnection structures are identical. Hence, we can say $(A, B)$ has the same structure as $(A 1, B 1)$, if for every fixed zero entry of the matrix $\left[\begin{array}{ll}A & B\end{array}\right]$, the corresponding entry of the matrix $\left[\begin{array}{ll}A 1 & B 1\end{array}\right]$ is fixed zero and vice versa.

Definition 5. The structural rank (srank) of a matrix is the maximum rank of all structurally equivalent matrices [30].

Theorem 6 (controllability test [31]). $\left(A_{n \times n}, B_{n \times m}\right)$ is controllable if and only if there is no left eigenvector of $A$ that is orthogonal to $B$; that is,

$$
\forall w \quad \text { where } w^{T} A=\lambda w^{T} \Longrightarrow w^{T} B \neq 0 .
$$

Theorem 7 (Popov-Belevitch-Hautus controllability test [32]). $\left(A_{n \times n}, B_{n \times m}\right)$ is controllable if and only if

$$
\operatorname{Rank}[\lambda I-A \quad B]=n,
$$

where $\lambda$ is an eigenvalue of $A$.

\section{Model of Interconnected Networks}

We consider each node in the network corresponding to a dynamical system, governed by the following equation:

$$
\dot{x}_{i}=-a_{i i} x_{i}+\sum_{\substack{j=1 \\ j \neq i}}^{N} a_{i j} x_{j}+b_{i} u_{i},
$$

where $x_{i} \in R$ denotes the state of node $i, N$ is the total number of nodes, and $u_{i}$ denotes an external input. form

Interconnected system (3) can be represented in matrix

$$
\dot{x}=A x+B u,
$$

where $x=\left[x_{1}, x_{2}, \ldots, x_{N}\right]^{T}, u=\left[u_{1}, u_{2}, \ldots, u_{m}\right]^{T}, B \in R^{N \times m}$ is input matrix, and $A \in R^{n \times n}$ is defined as $A=\mathscr{A}-\mathscr{D}$, where $\mathscr{A}$ is the adjacency matrix and $\mathscr{D}=\operatorname{diag}\left\{a_{11}, a_{22}, \ldots, a_{N N}\right\}$. 


\section{Controllability and Graph Reachability}

According to classical control theory, a dynamical system is controllable if for any initial state there exists an input that can drive the system to any final state in a finite time. It is well known that the system $(A, B)$ is controllable if and only if the following controllability matrix:

$$
\mathscr{C}=\left[B, A B, \ldots, A^{N-1} B\right]
$$

has full rank. Even though a system with a pair of $(A, B)$ might be uncontrollable, it can be controllable for another structurally equivalent pair $\left(A^{*}, B^{*}\right)[18]$.

Definition 8. A dynamical system $(A, B)$ is structurally controllable if there exists a structurally equivalent system $\left(A^{*}, B^{*}\right)$ that is controllable [33].

In what follows, we first consider network with single driver node and present the relation between graph reachability and controllability.

4.1. Controllability of Networks with Single Driver Node. Consider a network of nodes with single driver node, $i$, which is expressed as follows:

$$
\dot{x}=A x+B u,
$$

where $B=[\underbrace{0, \ldots, 0, b_{i}, 0, \ldots, 0}_{\text {ith element }}]^{T}$. To examine the controllability, we form the following zero-state response:

$$
x(t)=\int_{0}^{t} e^{A(t-\tau)} B u(\tau) d \tau .
$$

The term $e^{A(t-\tau)} B$ refers to the $i$ th column of the matrix $e^{A(t-\tau)}$ multiplied by $b_{i}$. Using Cayley-Hamilton theorem, $e^{A(t-\tau)} \mathrm{can}$ be expanded as follows:

$$
e^{A(t-\tau)}=\sum_{i=0}^{N-1} \alpha_{i}(t-\tau) A^{i},
$$

where $\alpha_{i}(\cdot)$ are scalar functions. We state the following theorem.

Theorem 9. Consider the network expressed by (6). The network is structurally controllable if and only if there is an arborescence subdigraph diverging from driver node $i$.

Proof (necessity condition). According to Lemma A.1 in the Appendix, the $(j, i)$ th element of matrix series (8) is zero, if there is no path from node $i$ to node $j$. In this case, the $j$ th element of the $e^{A(t-\tau)} B$ is zero and remains zero for all values of the network link weights; therefore at least $x^{*}=$ $[\underbrace{0, \ldots, 0, \alpha, 0, \ldots, 0}_{\text {jth element }}]^{T}$ is an uncontrollable state of the system.

Sufficiency Condition. We show that a network, which contains an arborescence subdigraph diverging from its driver node, is structurally controllable. Without loss of generality, we assume that node 1 is the driver node. In structural controllability, independent nonzero parameters can take any values including zero. Hence, we zero out the weights for redundant links in such a way that the digraph associated with the network becomes an arborescence diverging from the driver node.

Matrices $A$ and $B$ in (4) for an arborescence diverging from driver node can be expressed as follows:

$$
\begin{aligned}
A & =\left[\begin{array}{cccc}
-a_{11} & 0 & \cdots & 0 \\
a_{21} & -a_{22} & & \vdots \\
\vdots & \vdots & \ddots & 0 \\
a_{N 1} & a_{N 2} & \cdots & -a_{N N}
\end{array}\right], \\
B & =\left[\begin{array}{llll}
b_{1} & 0 & \cdots & 0
\end{array}\right]^{T} .
\end{aligned}
$$

Let $w_{i}=\left[w_{1}^{i}, \ldots, w_{N}^{i}\right]^{T}$ be the left eigenvector associated with eigenvalue $\lambda_{i}$. Using Theorem 6, we have

$$
w_{i}^{T} B \neq 0 \Longleftrightarrow w_{1}^{i} b_{1} \neq 0 \Longleftrightarrow w_{1}^{i} \neq 0 .
$$

Therefore, to show that the network is structurally controllable, we need to prove the existence of weights such that $w_{1}^{i} \neq 0$, for $i=1, \ldots, N$. To do so, let $a_{i i}$ be a strictly monotonic sequence for $i=1, \ldots, N$. Since $A$ is triangular matrix with distinct diagonal entries, eigenvalues of $A$ are its diagonal entries; that is, $\lambda_{i}=-a_{i i}$ for $i=1, \ldots, N$. Therefore, we obtain $w_{1}^{i}$ by solving $w_{i}^{T} A=\lambda_{i} w_{i}$ as follows:

$$
\begin{aligned}
& \lambda_{1}=-a_{11} \\
& \Longrightarrow w_{1}^{1}=1, \\
& \lambda_{2}=-a_{22} \\
& \Longrightarrow w_{1}^{2}=\frac{a_{21}}{a_{11}-a_{22}}, \\
& \lambda_{3}=-a_{33} \\
& \Longrightarrow w_{1}^{3}=\frac{a_{31}}{a_{11}-a_{33}}+\frac{a_{32} a_{21}}{\left(a_{22}-a_{33}\right)\left(a_{11}-a_{33}\right)} \\
& \lambda_{N}=-a_{N N} \\
& \Longrightarrow w_{1}^{N}=\frac{a_{N 1}}{a_{11}-a_{N N}}+\sum_{l_{1}=2}^{N-1} \frac{a_{N l_{1}} a_{l_{1} 1}}{\left(a_{l_{1} l_{1}}-a_{N N}\right)\left(a_{11}-a_{N N}\right)} \\
& +\sum_{l_{1}=3}^{N-1} \sum_{l_{2}=2} \frac{a_{N l_{1}} a_{l_{1} l_{2}} a_{l_{2} 1}}{\left(a_{l_{1} l_{1}}-a_{N N}\right)\left(a_{l_{2} l_{2}}-a_{N N}\right)\left(a_{11}-a_{N N}\right)} \\
& +\sum_{l_{1}=N-1}^{N-1} \ldots \sum_{N-1=2}^{l_{N-2}-1} \frac{\vdots}{\left(a_{l_{1} l_{1}}-a_{N N}\right) \cdots\left(a_{l_{N-1} l_{N-1}}-a_{N N}\right)\left(a_{11}-a_{N N}\right)} . \\
& a_{N l_{1} a_{l_{1}} l_{2} \cdots a_{l_{N-1} 1}}
\end{aligned}
$$


Since all the denominators of (11) have the same sign and $w_{1}^{i}$ for $i=1, \ldots, N$ can be expressed as

$$
\begin{aligned}
w_{1}^{i}= & \alpha_{1}^{1} a_{i 1}+\sum_{l_{1}=1}^{i-1} \alpha_{2}^{l_{1}} \underbrace{a_{i l_{1}} a_{l_{1} 1}}_{\begin{array}{c}
\text { path of length } \\
2 \text { from } 1 \text { to } i
\end{array}} \\
& +\sum_{l_{1}=1}^{i-1} \sum_{l_{2}=1}^{l_{1}-1} \alpha_{3}^{l_{1} l_{2}} \underbrace{a_{i l_{1}} a_{l_{1} l_{2}} a_{l_{2} 1}}_{\begin{array}{c}
\text { path of length } \\
3 \text { from } 1 \text { to } i
\end{array}}+\cdots \\
& +\sum_{l_{1}=i-1}^{i-1} \cdots \sum_{l_{i-1}=2}^{l_{i-2}-1} \alpha_{i-1}^{1} \underbrace{a_{i l_{1}} a_{l_{1} l_{2}} \cdots a_{l_{i-1} 1}}_{\begin{array}{c}
\text { path of length } \\
i-1 \text { from } 1 \text { to } i
\end{array}},
\end{aligned}
$$

where $\alpha_{k}^{l}$ are same sign scalars for different values of $k$ and $l$. Existence of arborescence diverging from node 1 guarantees that $w_{1}^{i} \neq 0$. Hence, $w^{i} B \neq 0$ for $i=1, \ldots, N$.

Since an arborescence diverging from the driver node is structurally controllable, we can conclude that any networked system which contains an arborescence subdigraph diverging from the driver node is structurally controllable.

Corollary 10. A network with a globally reachable driver node is structurally controllable.

In the above, we examine networks with single driver node. In what follows, we generalize the result for networks with multiple driver nodes.

4.2. Controllability of Networks with Multiple Driver Nodes. Consider a network of nodes with multiple driver nodes, which is expressed as follows:

$$
\dot{x}=A x+B u,
$$

where $B=r \operatorname{diag}\left\{b_{1}, b_{2}, \ldots, b_{m}\right\} \in R^{N \times m}$, where $r \operatorname{diag}\{\cdot\}$ refers to the rectangular diagonal matrix and $b_{r}$ are positive scalars. The following theorem expresses the controllability condition in networks with multiple driver nodes.

Theorem 11. Consider the network expressed by (13) which consists of multiple driver nodes. The network is structurally controllable if and only if there is a path from at least one driver node to any arbitrary node.

Proof. For simplicity, we assume that nodes $i=1, \ldots, m$ are driver nodes of the network. Hence matrix $B$ can be expressed as follows:

$$
B=\left[\begin{array}{cccc}
b_{1} & 0 & \cdots & 0 \\
0 & b_{2} & & \vdots \\
\vdots & & \ddots & 0 \\
0 & \cdots & 0 & b_{m} \\
& \mathbf{0}_{(N-m) \times m} &
\end{array}\right]
$$

Necessity Condition. We assume that there is node $j$ and that there is no path from any input node to that node. According to Lemma A.1 in the Appendix, matrix series (8) has zero elements in columns 1 to $m$ of row $j$. Therefore, $e^{A(t-\tau)} B$ has zero row $j$, which yields existence of $x^{*}=$ $[\underbrace{0, \ldots, 0, \alpha, 0, \ldots, 0}]^{T}$ as an uncontrollable state of the system. $j$ th element

Sufficiency Condition. We assume that the network contains $m$ driver nodes. By zeroing out the weights of redundant links, we decompose the network into $m$ components such that driver node $k$ controls over nodes of component $k$. Hence, matrices $A$ and $B$ can be expressed as follows:

$$
\begin{gathered}
A=\left[\begin{array}{lll}
A_{1} & & \mathbf{0} \\
& \ddots & \\
\mathbf{0} & & A_{m}
\end{array}\right], \\
B=\left[\begin{array}{lll}
B_{1} & & \mathbf{0} \\
& \ddots & \\
\mathbf{0} & & B_{m}
\end{array}\right] .
\end{gathered}
$$

If $l$ th node in $k$ th component is driver node, we have $B_{k}=$ $[\underbrace{0, \ldots, 0, b_{k}, 0, \ldots, 0}]^{T}$ for $k=1, \ldots, N$. Using Theorem 7 , we obtain

$$
\begin{aligned}
& \operatorname{Rank}[\lambda I-A \quad B] \\
& \quad=\operatorname{Rank}\left[\begin{array}{ccccc}
\lambda I_{1}-A_{1} & B_{1} & & & 0 \\
& & \ddots & & \\
0 & & & \lambda I_{m}-A_{m} & B_{m}
\end{array}\right],
\end{aligned}
$$

where $I_{k}$ are identity matrices with the same size as $A_{k}$ for $k=1, \ldots, N$. Using Theorem 9 , for an arbitrary component $k,\left[\begin{array}{ll}\lambda I_{k}-A_{k} & B_{k}\end{array}\right]$ is full row-rank. Since $[\lambda I-A B]$ is block diagonal matrix with full row-rank block matrices, therefore

$$
\operatorname{Rank}\left[\begin{array}{ll}
\lambda I-A & B
\end{array}\right]=N .
$$

For better underdressing, in what follows, we compare the proposed structural controllability condition and Liu's structural controllability condition. An example of Liu's structural controllability is presented in Figure 1. It is shown that controlling node 1 is not sufficient for full control (see Figure 1(a)). To gain full control, we must simultaneously control node 1 and any node among $x_{2}, x_{3}$ (see Figure 1(b)). In contrast, in the proposed structural controllability (see Figure 2), controlling node 1 is sufficient for full control over the networked system.

\section{Algorithm for Identifying Minimum Driver Nodes}

We have shown the relationship between the structural controllability and graph reachability. Thus the problem of 


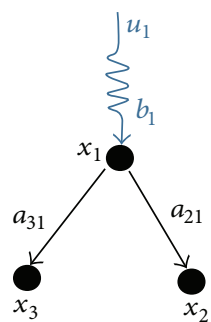

$$
\begin{gathered}
A=\left[\begin{array}{ccc}
0 & 0 & 0 \\
a_{21} & 0 & 0 \\
a_{31} & 0 & 0
\end{array}\right] ; B=\left[\begin{array}{c}
b_{1} \\
0 \\
0
\end{array}\right] ; \mathscr{C}=\left[\begin{array}{cccc}
b_{1} & 0 & 0 & 0 \\
0 & b_{1} a_{21} & 0 & 0 \\
0 & b_{1} a_{31} & 0 & 0
\end{array}\right] \\
n=3, m=1, \operatorname{srank}(\mathscr{C})=2
\end{gathered}
$$

(a)

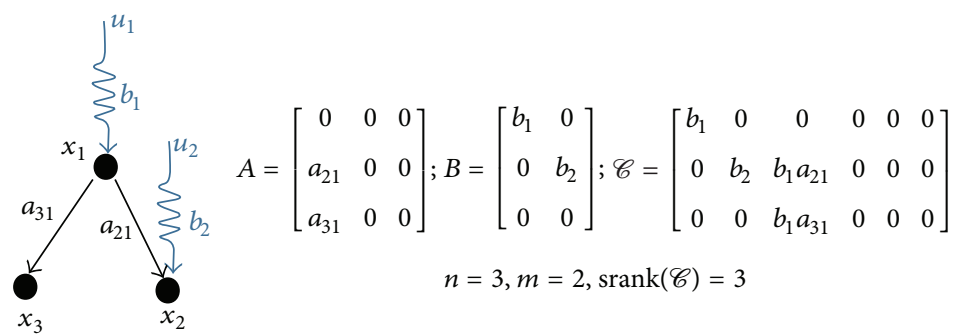

(b)

FIgURE 1: An example of Liu's structural controllability.

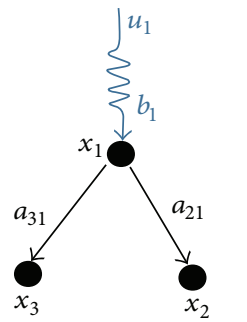

$$
\begin{gathered}
A=\left[\begin{array}{ccc}
a_{11} & 0 & 0 \\
a_{21} & a_{22} & 0 \\
a_{31} & 0 & a_{33}
\end{array}\right] ; B=\left[\begin{array}{c}
b_{1} \\
0 \\
0
\end{array}\right] ; \mathscr{C}=\left[\begin{array}{ccc}
b_{1} & b_{1} a_{11} & b_{1} a_{11}^{2} \\
0 & b_{1} a_{21} & b_{1} a_{21}\left(a_{11}+a_{22}\right) \\
0 & b_{1} a_{31} & b_{1} a_{31}\left(a_{11}+a_{33}\right)
\end{array}\right] \\
N=3, m=1, \operatorname{srank}(\mathscr{C})=3
\end{gathered}
$$

FIgURE 2: An example of the proposed structural controllability.

examining the structural controllability of the networked systems described by (5) can be converted into graph reachability problem. Here, we are interested in determining the minimum number of driver nodes in a directed network, denoted by $N_{D}$, to obtain controllability over the networked systems. However, difficulties in identifying minimum number of driver nodes in large-scale networks lead to the requirement for a simple systematic method. In what follows, we propose a simple algorithm to determine the minimum number of driver nodes using graph reachability approach.

To check the graph reachability between each two arbitrary nodes, we present the following theorem.

Theorem 12. Consider a network of nodes with an associated structured adjacency matrix $\mathscr{A}_{s}$. For any two arbitrary nodes $i$ and $j$, if $(j, i)$ th element of the matrix $\left(\mathbf{I}-\mathscr{A}_{s} t\right)^{-1}$ is zero, then there is no path from node $i$ to node $j$, where $\mathbf{I}$ is an identity matrix and $t$ is a positive constant such that the spectral radius of $\mathscr{A}_{s} t$ is less than 1 .

Proof. To prove this theorem, we first use the Taylor series expansion of the matrix inverse (see Lemma A.3 in the Appendix). Consider

$$
\left(\mathbf{I}-\mathscr{A}_{s} t\right)^{-1}=\sum_{i=0}^{\infty} t^{i} \mathscr{A}_{s}^{i} .
$$

Using Lemma A.2, $\left(\mathscr{A}_{s}^{k}\right)_{j i}$ for $k=1,2, \ldots, \infty$ is zero if and only if there is no path from node $i$ to node $j$. Hence, the zeroness of the $(j, i)$ th entry of $\mathscr{A}_{s}^{k}$ for $k=1,2, \ldots, \infty$ leads to the zeroness of the $(j, i)$ th entry of $\left(\mathbf{I}-\mathscr{A}_{s} t\right)^{-1}$.

Remark 13. Using Gershgorin's theorem [34], the suitable $t$ which satisfies the condition in Theorem 12 is obtained as follows:

$$
t=\left(\max _{\substack{i \\ j}} \sum_{\substack{j=1 \\ j \neq i}}^{N} a_{i j}\right)^{-1}-\varepsilon,
$$

where $\varepsilon$ is a small number.

To illustrate the result in Theorem 12, let us consider the network in Figure 3.

The associated structured matrix can be defined as Boolean matrix as follows:

$$
\mathscr{A}_{s}=\left[\begin{array}{llll}
0 & 1 & 1 & 1 \\
0 & 0 & 0 & 1 \\
1 & 1 & 0 & 1 \\
0 & 1 & 0 & 0
\end{array}\right] .
$$




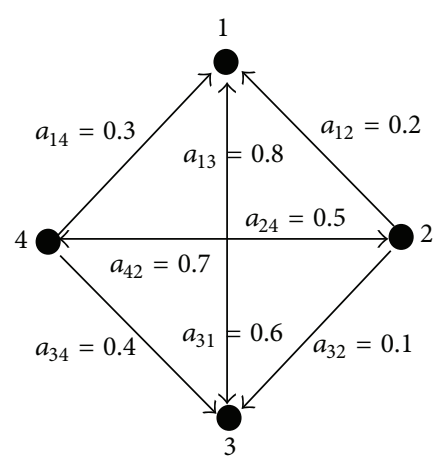

FIgURE 3: An example of a network with 4 nodes.

From (19), we obtain $t=0.1$. Therefore, matrix $\left(\mathbf{I}-\mathscr{A}_{s} t\right)^{-1}$ is obtained in structured format as follows:

$$
\left[\begin{array}{llll}
* & * & * & * \\
0 & * & 0 & * \\
* & * & * & * \\
0 & * & 0 & *
\end{array}\right],
$$

where $*$ represents nonzero parameters such that in matrix (21), for example, entry $(4,1)$ is zero, which means that there is no path from node 1 to node 4 . Since the network is small, driver nodes in Figure 3 can be easily identified, which are either node 2 or node 4 . The same result can be obtained by examining $\left(\mathbf{I}-\mathscr{A}_{s} t\right)^{-1}$. In matrix (21), columns full of nonzero elements represent globally reachable nodes. For columns which contain zero elements, we define graph reachability index as follows.

Definition 14. Node $u$ is said to have graph reachability index $r$, if there are paths from $u$ to maximum $r$, other nodes of the network.

Therefore, we can express the following corollary.

Corollary 15. In matrix $(\mathbf{I}-\mathscr{A} t)^{-1}$, columns with higher nonzero elements represent nodes with higher graph reachability index.

We can deduce that nodes with higher graph reachability index are suitable to be assigned as driver nodes.

Remark 16. To find the minimum driver nodes to obtain a structurally controllable network, we start by assigning the node with the highest graph reachability index as the driver node. Then, we remove all the nodes that are in the path rooted for the assigned driver node. We repeat the above procedure for the remaining network till the condition in Theorem 11 is satisfied.

Using the above mentioned results, we present a systematic algorithm to identify the minimum driver nodes in a networked system such that the structural controllability of the network is guaranteed. The algorithm for determining the minimum driver nodes of the network is described as follows.

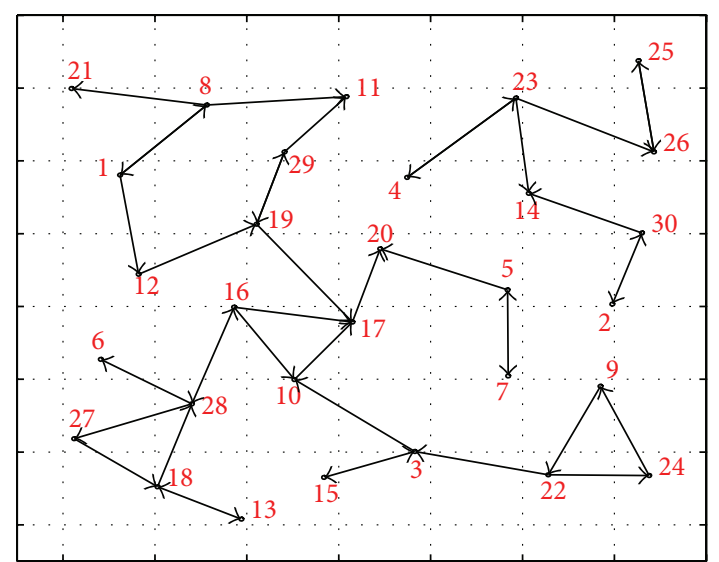

FIgURE 4: An example of a network consisting of 30 nodes.

Consider graph $\mathscr{G}$ with the associated structured adjacency matrix $\mathscr{A}_{s}$,

Step 1. Compute graph reachability matrix $\mathcal{S}=\left(\mathbf{I}_{N}-\mathscr{A}_{s} t\right)^{-1}$.

Step 2. Identify the node with the highest graph reachability index by finding the columns of matrix $\mathcal{S}$ with the largest nonzero elements. If there is more than one node with the highest graph reachability index, we can randomly choose one of them.

Step 3. Assign that node as the driver node and zero out all the rows with the nonzero elements in the column associated with that driver node.

Step 4. Go back to Step 2 and repeat the procedure till all elements of matrix $\mathcal{S}$ are zero.

The above procedure is expressed in Algorithm 1.

Remark 17. It should be noted that the set of minimum driver nodes is usually not unique depending on the network configurations, and one can determine other sets with the same number of driver nodes.

\section{Simulations}

In this section, we present simulation results to illustrate the performance of the proposed method for networked systems of various sizes and topologies. For the numerical calculations and simulations, we used MATLAB software. For illustration purpose, we first consider a network with 30 nodes which are distributed randomly as depicted in Figure 4. The weights of links are randomly selected from $[0,1]$. We compute $(\mathbf{I}-\mathscr{A} t)^{-1}$, where $\mathscr{A}$ is the associated Laplacian matrix. The sparsity pattern of matrix $(\mathbf{I}-\mathscr{A} t)^{-1}$ is plotted in Figure 5, where the blue solid circles represent nonzero elements of the matrix. Applying the proposed algorithm, the driver nodes of the network are identified by magenta circles in Figure 6. The result of the first simulation is summarized in Table 1 , where $N$ is the number of nodes, $L$ is the number 


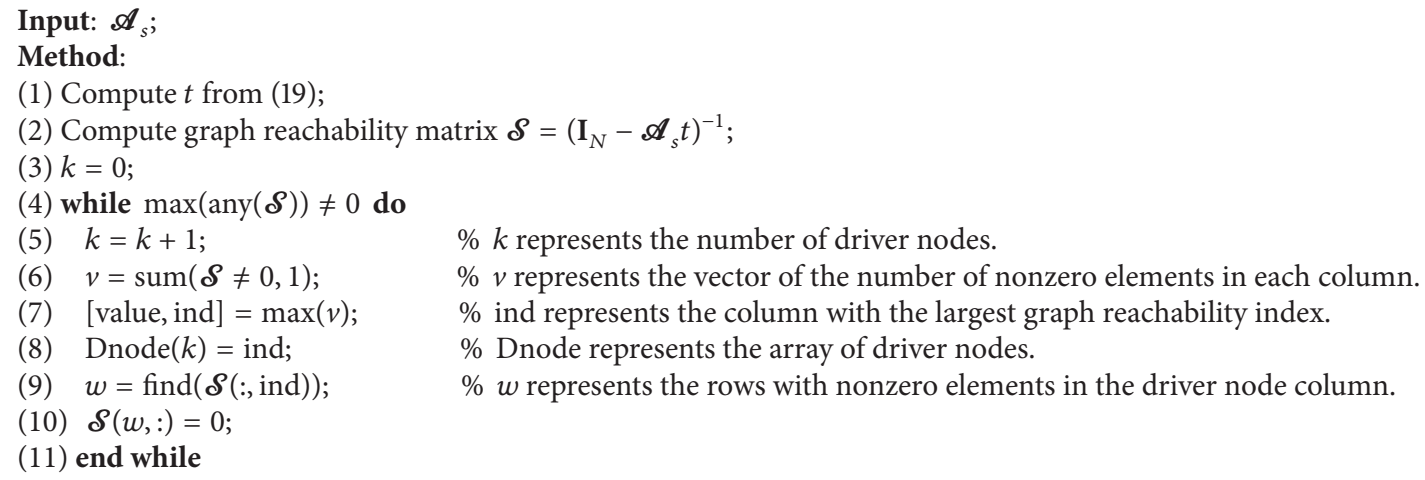

$\% k$ represents the number of driver nodes.

$\% v$ represents the vector of the number of nonzero elements in each column.

$\%$ ind represents the column with the largest graph reachability index.

$\%$ Dnode represents the array of driver nodes.

$\% w$ represents the rows with nonzero elements in the driver node column.

Algorithm 1: Finding driver nodes in each connected component.

TABLE 1: The characteristics of the network represented in Figure 6.

\begin{tabular}{llcc}
\hline$N$ & $L$ & $N_{D}$ & $n_{d}$ \\
\hline 30 & 41 & 6 & 0.2 \\
\hline
\end{tabular}

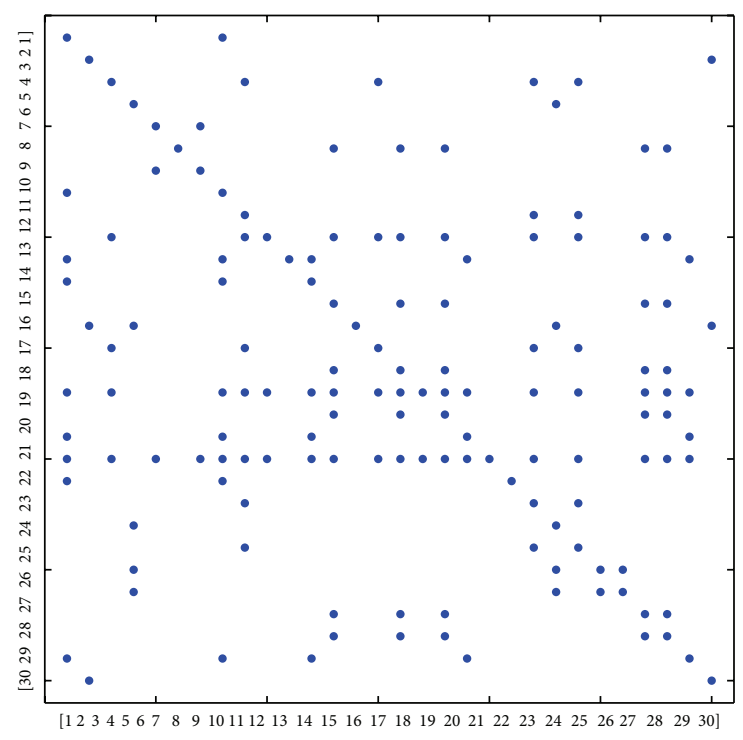

FIgURE 5: The sparsity pattern of matrix $(\mathbf{I}-\mathscr{A} t)^{-1}$.

of links, $N_{D}$ is the computed number of driver nodes, and $n_{d}$ is the computed density of driver nodes obtained by $n_{d}=$ $N_{d} / N$.

To illustrate the capability of the purposed algorithm in dealing with large-scale networks, we consider a network of 1000 nodes which are distributed randomly within a square region as shown in Figure 7 . The communication links are generated between neighboring nodes with the probability of 0.5 . The weights of links are randomly selected from $[0,1]$. The sparsity pattern of matrix $(\mathbf{I}-\mathscr{A} t)^{-1}$ is plotted for the network in Figure 8. Applying the proposed algorithm, the driver nodes of the network are identified by magenta circles in Figure 9. The result of the second simulation is summarized in Table 2.

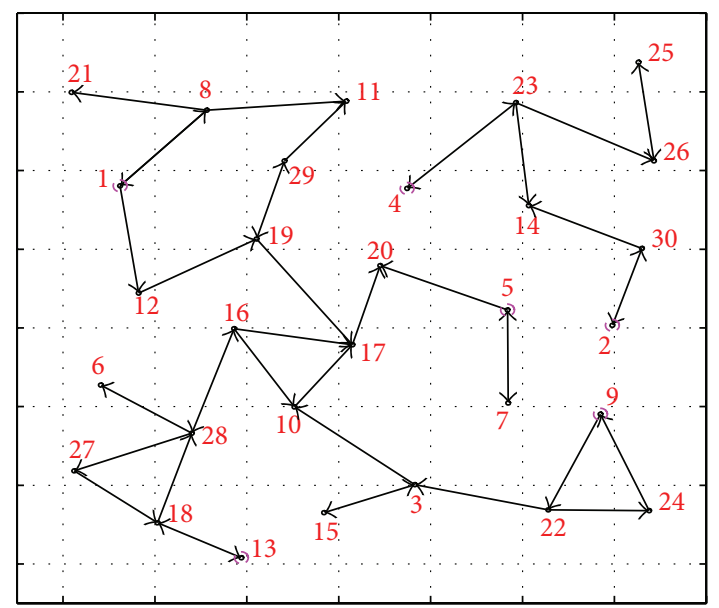

Figure 6: Driver nodes of the network identified by magenta circles.

TABLE 2: The characteristics of the network represented in Figure 9.

\begin{tabular}{lccc}
\hline$N$ & $L$ & $N_{D}$ & $n_{d}$ \\
\hline 1000 & 1361 & 96 & 0.096 \\
\hline
\end{tabular}

TABLE 3: The characteristics of some randomly generated networks.

\begin{tabular}{lccc}
\hline$N$ & $L$ & $N_{D}$ & $n_{d}$ \\
\hline 2000 & 2929 & 128 & 0.0640 \\
5000 & 6931 & 408 & 0.0816 \\
10000 & 13951 & 748 & 0.0748 \\
\hline
\end{tabular}

We applied the proposed algorithm on some randomly generated networks, and the results are illustrated in Table 3.

\section{Conclusion}

In this paper, we have addressed the structural controllability problem for networked systems. Despite the existing methods governed by some impractical assumptions on nodal dynamics and availability of input signals, we have examined structural controllability for networked systems in 


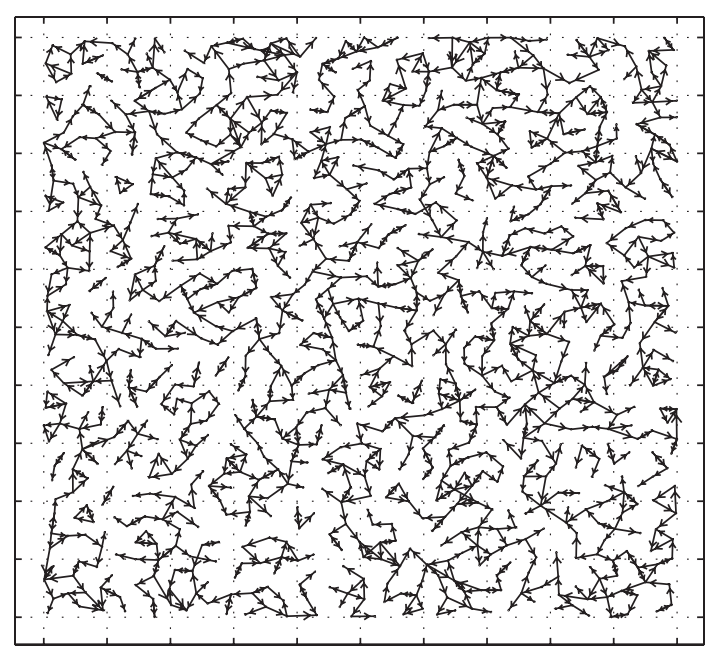

FIGURE 7: An example of a network consisting of 1000 nodes.

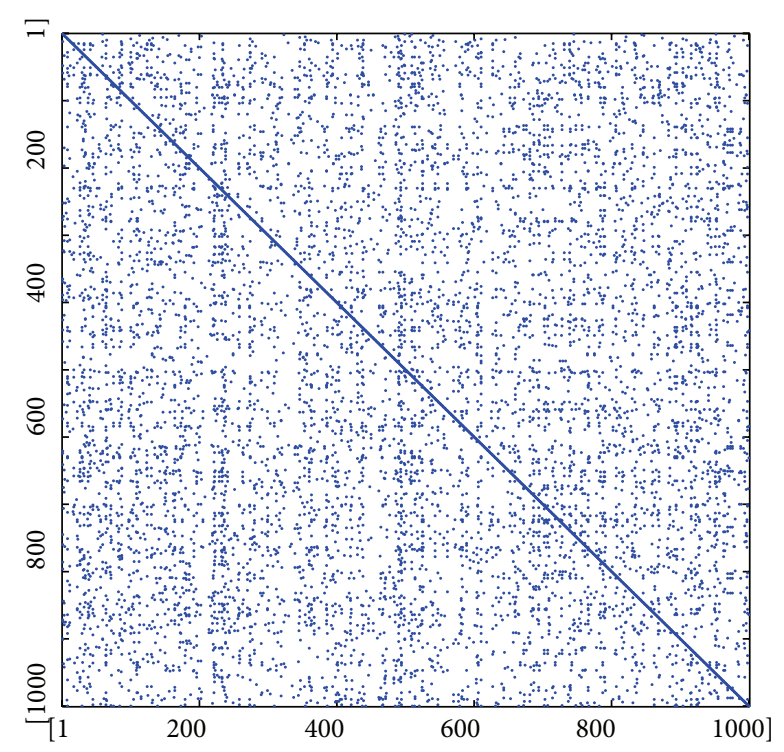

FIgURE 8: The sparsity pattern of matrix $(\mathbf{I}-\mathscr{A} t)^{-1}$.

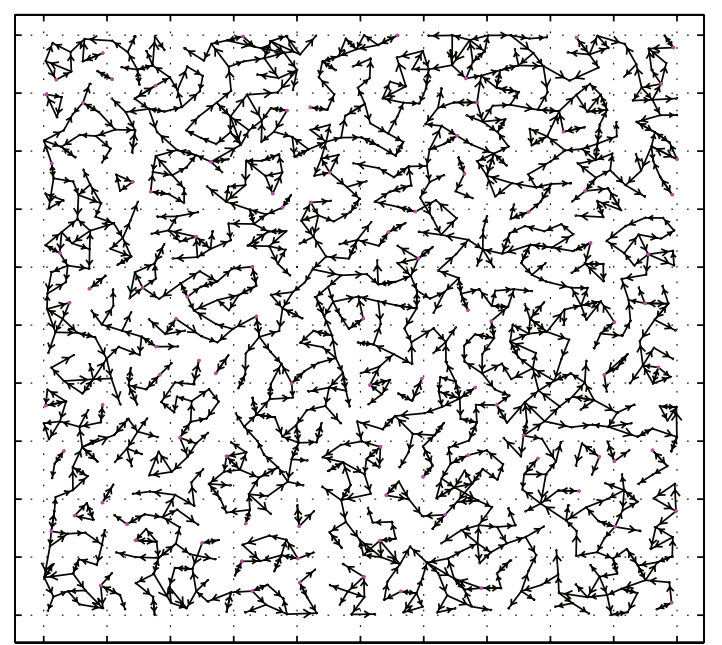

FIGURE 9: Driver nodes of the network identified by magenta circles. practical framework. Using controllability analysis, we have presented the connection between networks driver nodes and graph reachability. Consequently, based on results on graph reachability, we have put forward a simple algorithm to determine minimum driver nodes in networked systems. Finally, simulation results have been presented to illustrate the performance of the proposed methods.

\section{Appendix}

Lemma A.1. Let $\mathscr{L}=\mathscr{A}-\mathscr{D}$ where $\mathscr{A}$ is the adjacency matrix and $\mathscr{D}=\operatorname{diag}\left\{a_{11}, a_{22}, \ldots, a_{N N}\right\}$. Consider the following matrix:

$$
\mathscr{P}=\sum_{i=1}^{N-1} \beta_{i} \mathscr{L}^{i}
$$

where $\beta_{i}$ are scalars. $(\mathscr{P})_{i j}$ is zero for any arbitrary values of $\beta_{i}$, if there is no path of any length from node $j$ to node $i$.

Proof. To prove the lemma, we show that the $(i, j)$ th element of all matrices $\mathscr{L}^{i}$ where $i=1,2, \ldots, N$ is zero, if there is no path of any length from node $j$ to node $i$. Since there is no adjacent path from node $j$ to $i$, then $a_{i j}=0$. Therefore, the $(i, j)$ th element of the $\mathscr{L}^{2}$ can be expressed as follows:

$$
\left(\mathscr{L}^{2}\right)_{i j}=\sum_{\substack{k_{1}=1 \\ k_{1} \neq j}}^{N} a_{i k_{1}} a_{k_{1} j}=\left(\mathscr{A}^{2}\right)_{i j}
$$

Using Lemma A.2, we obtain $\left(\mathscr{L}^{2}\right)_{i j}=0$. Therefore, the $(i, j)$ th element of the $\mathscr{L}^{3}$ can be expressed as follows:

$$
\left(\mathscr{L}^{3}\right)_{i j}=\sum_{\substack{k_{1}=1 \\ k_{1} \neq i, j}}^{N} \sum_{\substack{k_{2}=1 \\ k_{2} \neq i, j}}^{N} a_{i k_{1}} a_{k_{1} k_{2}} a_{k_{2} j}=\left(\mathscr{A}^{3}\right)_{i j} .
$$

Using Lemma A.2, we obtain $\left(\mathscr{L}^{3}\right)_{i j}=0$. Similarly, we can proceed for $\mathscr{L}^{4}, \mathscr{L}^{5}, \ldots, \mathscr{L}^{N-1}$ and show that $\left(\mathscr{L}^{k}\right)_{i j}=0$ for $k=1,2, \ldots, N-1$.

Lemma A.2 (see [35]). Let $\mathscr{A}$ be the adjacency matrix of a digraph $\mathscr{G}$; then $\left(\mathscr{A}^{k}\right)_{i j}$ is greater than zero if and only if there is a path of length $k$ from node $j$ to node $i$.

Lemma A.3. For two arbitrary matrices $A$ and B, the Taylor series expansion of the matrix inverse is expressed as follows:

$$
(A+B)^{-1}=A^{-1} \sum_{i=0}^{\infty}(-1)^{i}\left(B A^{-1}\right)^{i}
$$

where the spectral radius of $B A^{-1}$ is less than 1 .

\section{Conflict of Interests}

The authors declare that there is no conflict of interests regarding the publication of this paper. 


\section{References}

[1] H. G. Tanner, "On the controllability of nearest neighbor interconnections," in Proceedings of the 43rd IEEE Conference on Decision and Control (CDC '04), vol. 3, pp. 2467-2472, December 2004.

[2] M. Ji, A. Muhammad, and M. Egerstedt, "Leader-based multiagent coordination: controllability and optimal control," in Proceedings of the American Control Conference, pp. 1358-1363, June 2006.

[3] A. Rahmani and M. Mesbahi, "On the controlled agreement problem," in Proceedings of the American Control Conference, pp. 1376-1381, IEEE, Minneapolis, Minn, USA, June 2006.

[4] M. Ji and M. Egerstedt, "A graph-theoretic characterization of controllability for multi-agent systems," in Proceedings of the American Control Conference (ACC '07), pp. 4588-4593, IEEE, New York, NY, USA, July 2007.

[5] A. Rahmani, M. Ji, M. Mesbahi, and M. Egerstedt, "Controllability of multi-agent systems from a graph-theoretic perspective," SIAM Journal on Control and Optimization, vol. 48, no. 1, pp. 162-186, 2009.

[6] B. Liu, T. Chu, L. Wang, and G. Xie, "Controllability of a leader-follower dynamic network with switching topology," IEEE Transactions on Automatic Control, vol. 53, no. 4, pp. 10091013, 2008.

[7] B. Liu, T. Chu, L. Wang, Z. Zuo, G. Chen, and H. Su, "Controllability of switching networks of multi-agent systems," International Journal of Robust and Nonlinear Control, vol. 22, no. 6, pp. 630-644, 2012.

[8] Z. J. Ji, Z. D. Wang, H. Lin, and Z. Wang, "Interconnection topologies for multi-agent coordination under leader-follower framework," Automatica, vol. 45, no. 12, pp. 2857-2863, 2009.

[9] K. Peng and Y. Yang, "Leader-following consensus problem with a varying-velocity leader and time-varying delays," Physica $A$, vol. 388, no. 2-3, pp. 193-208, 2009.

[10] W. Ni and D. Cheng, "Leader-following consensus of multiagent systems under fixed and switching topologies," Systems \& Control Letters, vol. 59, no. 3-4, pp. 209-217, 2010.

[11] L. Consolini, F. Morbidi, D. Prattichizzo, and M. Tosques, "Leader-follower formation control of nonholonomic mobile robots with input constraints," Automatica, vol. 44, no. 5, pp. 1343-1349, 2008.

[12] R. Haghighi and C. C. Cheah, "On leader-based shape coordination," in Proceedings of the 11th International Conference on Control, Automation, Robotics \& Vision (ICARCV '10), pp. 404409, IEEE, Singapore, December 2010.

[13] R. Haghighi and C. C. Cheah, "Multi-group coordination control for robot swarms," Automatica, vol. 48, no. 10, pp. 25262534, 2012.

[14] H. Su, G. Jia, and M. Z. Q. Chen, "Semi-global containment control of multi-agent systems with input saturation," IET Control Theory \& Applications, vol. 8, no. 18, pp. 2229-2237, 2014.

[15] H. Su and M. Z. Q. Chen, "Multi-agent containment control with input saturation on switching topologies," IET Control Theory \& Applications, vol. 9, no. 3, pp. 399-409, 2015.

[16] M. Porfiri and M. di Bernardo, "Criteria for global pinningcontrollability of complex networks," Automatica, vol. 44, no. 12, pp. 3100-3106, 2008.

[17] Q. Song and J. Cao, "On pinning synchronization of directed and undirected complex dynamical networks," IEEE Transactions on Circuits and Systems I: Regular Papers, vol. 57, no. 3, pp. 672-680, 2010.
[18] C.-T. Lin, "Structural controllability," IEEE Transactions on Automatic Control, vol. 19, no. 3, pp. 201-208, 1974.

[19] R. W. Shields and J. B. Pearson, "Structural controllability of multi-input linear systems," IEEE Transactions on Automatic Control, vol. AC-21, no. 2, pp. 203-212, 1976.

[20] S. Hosoe and K. Matsumoto, "On the irreducibility condition in the structural controllability theorem," IEEE Transactions on Automatic Control, vol. 24, no. 6, pp. 963-966, 1979.

[21] J.-M. Dion, C. Commault, and J. van der Woude, "Generic properties and control of linear structured systems: a survey," Automatica, vol. 39, no. 7, pp. 1125-1144, 2003.

[22] H. Mayeda, "On structural controllability theorem," IEEE Transactions on Automatic Control, vol. 26, no. 3, pp. 795-798, 1981.

[23] Y.-Y. Liu, J.-J. Slotine, and A.-L. Barabási, "Controllability of complex networks," Nature, vol. 473, no. 7346, pp. 167-173, 2011.

[24] S. Jafari, A. Ajorlou, and A. G. Aghdam, "Leader localization in multi-agent systems subject to failure: a graph-theoretic approach," Automatica, vol. 47, no. 8, pp. 1744-1750, 2011.

[25] S. Sundaram and C. N. Hadjicostis, "Structural controllability and observability of linear systems over finite fields with applications to multi-agent systems," IEEE Transactions on Automatic Control, vol. 58, no. 1, pp. 60-73, 2013.

[26] R. Haghighi and C. C. Cheah, "Distributed average consensus based on structural weight-balanceability," IET Control Theory \& Applications, vol. 9, no. 2, pp. 176-183, 2015.

[27] N. J. Cowan, E. J. Chastain, D. A. Vilhena, J. S. Freudenberg, and C. T. Bergstrom, "Nodal dynamics, not degree distributions, determine the structural controllability of complex networks," PLoS ONE, vol. 7, no. 6, Article ID e38398, 2012.

[28] W. Tutte, Graph Theory, Addison-Wesley, 1984.

[29] D. D. Siljak, Decentralized Control of Complex Systems, Academic Press, New York, NY, USA, 1991.

[30] K. J. Reinschke, Multivariable Control: A Graph-Theoretic Approach, Springer, 1988.

[31] P. R. Belanger, Control Engineering: A Modern Approach, Saunders College Publishing, 1995.

[32] R. L. Williams and D. A. Lawrence, Linear State-Space Control Systems, John Wiley \& Sons, 2007.

[33] C. Sueur and G. Dauphin-Tanguy, "Bond-graph approach for structural analysis of MIMO linear systems," Journal of the Franklin Institute, vol. 328, no. 1, pp. 55-70, 1991.

[34] R. S. Varga, Gershgorin and His Circles, Springer, Berlin, Germany, 2004.

[35] G. Williams, Linear Algebra with Applications, Jones and Bartlett Publishers, 2012. 


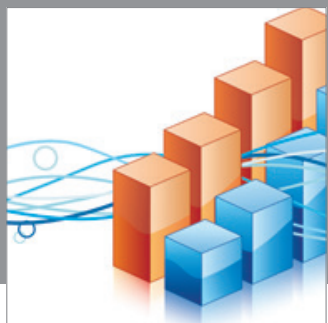

Advances in

Operations Research

mansans

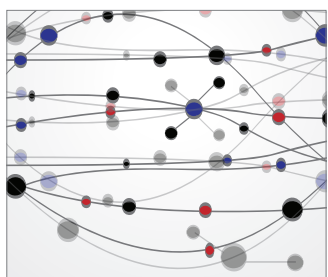

The Scientific World Journal
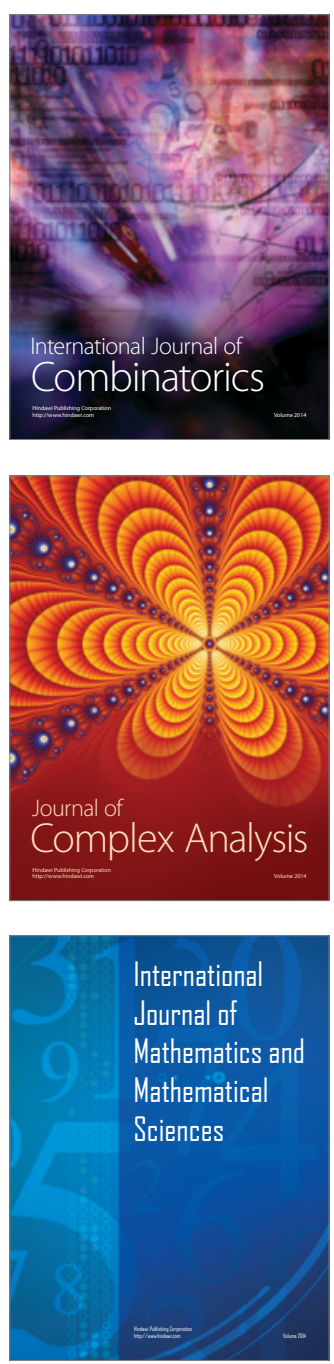
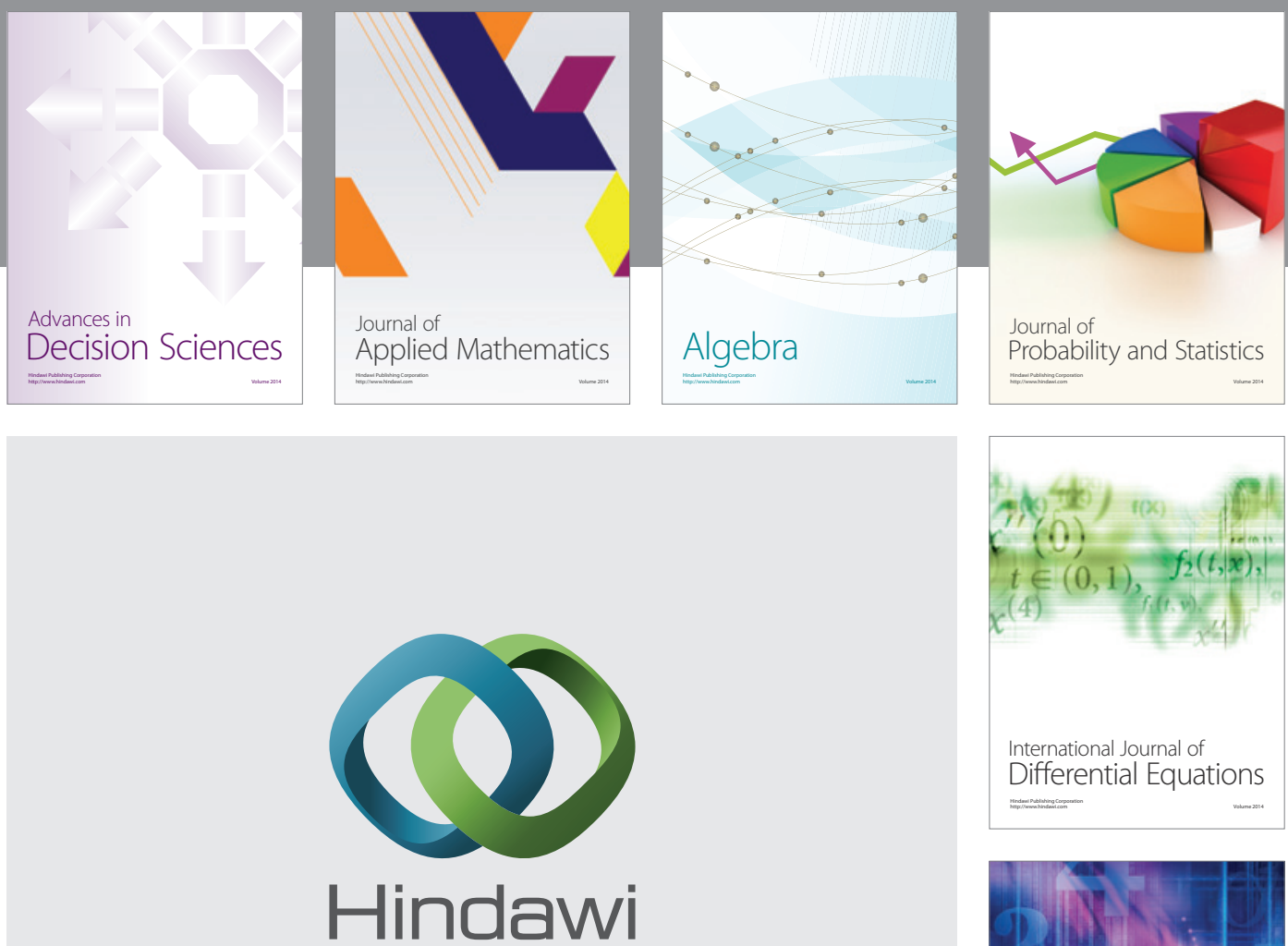

Submit your manuscripts at http://www.hindawi.com
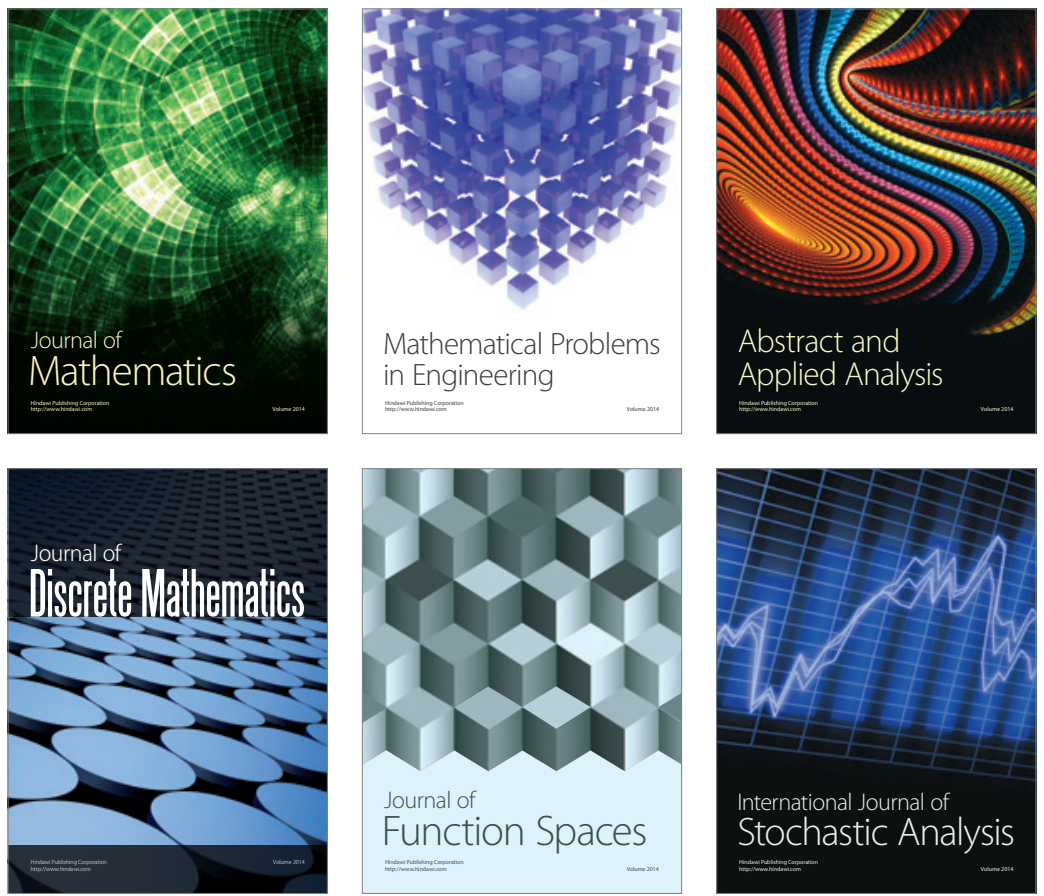

Journal of

Function Spaces

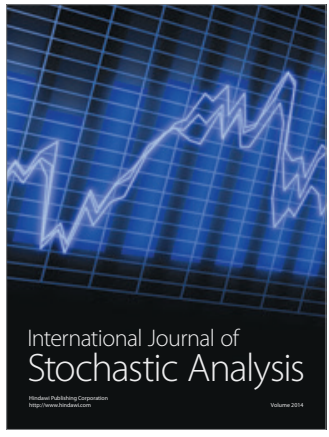

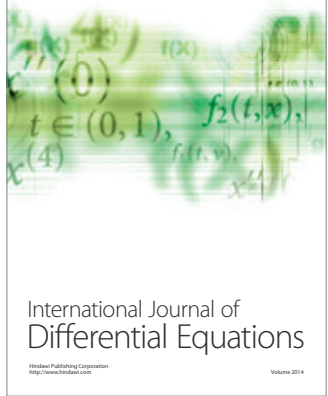
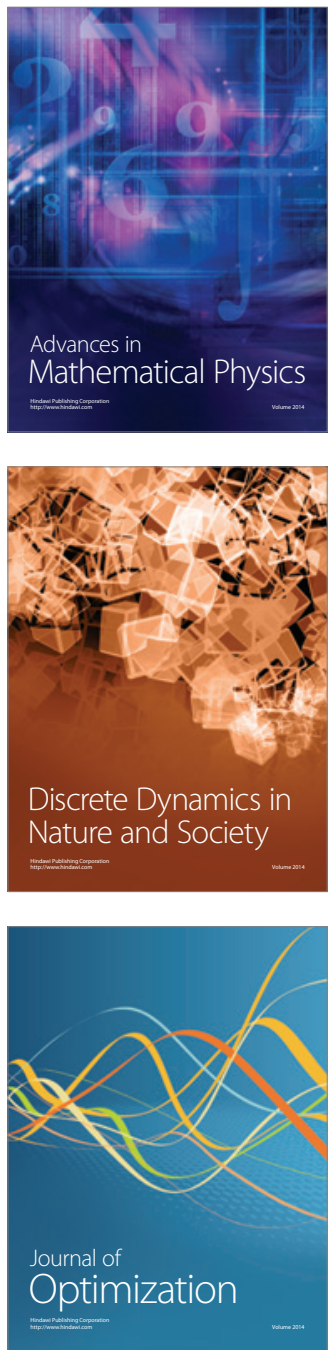